\title{
Über eine bemerkenswerte Eigenschaft von Dezimal- brüchen und gewissen anderen Systembrüchen
}

\author{
Stefan Deschauer \\ Stefan Deschauer studierte Mathematik, katholische Theologie und Erziehungswis- \\ senschaften an der Universität Marburg. Nach der Promotion in Ulm und einigen Jah- \\ ren im Schuldienst war er an den Universitäten Eichstätt und Erlangen tätig, bevor \\ er 1994 den Ruf auf eine Professur für Didaktik der Mathematik an der TU Dresden \\ annahm.
}

Während eines Unterrichtsbesuchs in einer 7. Klasse eines Dresdner Gymnasiums sollte eine Schülerin den Dezimalbruch 2,5 in einen gemeinen Bruch verwandeln. Ihre spontane Antwort war,$\frac{2}{5}$ “ , dann korrigierte sie und gab,$\frac{5}{2}$ “ an. Für den Mathematiker ergab sich sogleich folgende Frage:

Gibt es weitere endliche Dezimalbrüche, deren Wert mit dem Quotienten aus der natürlichen Zahl, die aus den Nachkommastellen gebildet wird, und der Vorkommazahl übereinstimmt?

$\mathrm{Zu}$ welchen Ergebnissen führt die entsprechende Untersuchung bei g-adischen Systembrüchen?

Der Reiz dieses keineswegs trivialen Problems besteht darin, dass die Fragestellung eigentlich so naheliegend ist, wichtige Teillösungen mit Methoden der elementaren Zahlentheorie gewonnen werden können und genügend offene Fragen bleiben.

Eine fehlerhafte Umwandlung von 2,5 (in $\frac{2}{5}$ ) im Unterricht machte den Autor auf die augenfällige Darstellung 2,5 $=\frac{5}{2}$ aufmerksam. Gibt es im Dezimalsystem noch weitere solcher Darstellungen, wobei die (ein- oder mehrstellige) Vorkommazahl mit dem Nenner und die (ein- oder mehrstellige) Nachkommazahl mit dem Zähler des gemeinen Bruchs übereinstimmt? Der elementare, aber attraktive Weg zur (negativen) Antwort weckt Neugier auf die Situation in anderen $g$-adischen Systemen. Ohne Weiteres findet man unendlich viele $g$ mit mindestens einer Darstellung (in obigem Sinne). Schwieriger ist es zu zeigen, dass sogar unendlich viele $g$ mit mindestens zwei Darstellungen existieren, und diese explizit anzugeben. 


\section{Dezimalbrüche}

Wir betrachten also allgemein Dezimalbrüche

$$
a, a_{n} a_{n-1} \ldots a_{1} a_{0}, \quad a \in \mathbb{N}^{*}, \quad 0 \leq a_{v} \leq 9 \quad(0 \leq v \leq n)
$$

mit der Eigenschaft

$$
a, a_{n} a_{n-1} \ldots a_{1} a_{0}=\frac{z}{a}, \quad z=\sum_{\nu=0}^{n} a_{v} \cdot 10^{v}<10^{n+1}
$$

Es folgt nach Multiplikation mit $10^{n+1} \cdot a$ :

$$
10^{n+1} \cdot a^{2}+a z=10^{n+1} \cdot z
$$

mit $a^{2}+a<10^{n+1}$ wegen $10^{n+1} \cdot a^{2}=\left(10^{n+1}-a\right) z<\left(10^{n+1}-a\right) 10^{n+1}$.

\subsection{Analyse des Terms für $z$}

Wir können nun nach $z$ auflösen und den entstehenden Term studieren. Es gilt

$$
z=\frac{10^{n+1} \cdot a^{2}}{10^{n+1}-a}
$$

Eine Umformung des Terms für $z$ führt zu

$$
z=a^{2}+q, q=\frac{a^{3}}{10^{n+1}-a} \in \mathbb{N}^{*} \quad \text { mit } a^{2}<10^{n+1}-a=\frac{a^{3}}{q}, \text { also } q<a .^{1}
$$

Letzteres ergibt sich auch aus der Identität

$$
\frac{z}{10^{n+1}}=\frac{q}{a} .
$$

Gemäß (4) oder (5) betrachten wir die multiplikative Darstellung

$$
a\left(a^{2}+q\right)=10^{n+1} q, \quad q<a .
$$

Mit der Zerlegung

$$
q=2^{s} \cdot 5^{t} \cdot q^{\prime} \cdot q^{\prime \prime}, \quad s, t \geq 0, \quad\left(10, q^{\prime} \cdot q^{\prime \prime}\right)=1, \quad q^{\prime}\left|a, \quad q^{\prime \prime}\right|\left(a^{2}+q\right)
$$

- die Wahl von $q^{\prime}$ und $q^{\prime \prime}$ ist zunächst nicht notwendig eindeutig - ergeben sich die Darstellungen

$$
\begin{gathered}
a=2^{i} \cdot 5^{k} q^{\prime}, 0 \leq i \leq n+1+s, 0 \leq k \leq n+1+t \\
\text { mit } i+k \geq 1 \text { wegen } q<a \\
a^{2}+q=2^{n+1+s-i} \cdot 5^{n+1+t-k} \cdot q^{\prime \prime} .
\end{gathered}
$$

(Wiederum sind nicht beide Potenzen $=1$.)

\footnotetext{
${ }^{1}$ Insgesamt gilt $z=a^{2}+q<a^{2}+a<10^{n+1}$.
} 
Andererseits ist

$$
\frac{a^{2}+q}{q^{\prime \prime}}=\frac{2^{2 i} \cdot 5^{2 k} \cdot q^{\prime 2}+2^{s} \cdot 5^{t} \cdot q^{\prime} q^{\prime \prime}}{q^{\prime \prime}}=2^{2 i} \cdot 5^{2 k} \cdot \frac{q^{\prime 2}}{q^{\prime \prime}}+2^{s} \cdot 5^{t} \cdot q^{\prime},
$$

so dass wegen der Bedingung der Teilerfremdheit in (7) gilt:

$$
q^{\prime \prime} \mid q^{\prime 2} \text {. }
$$

Darüber hinaus ergibt sich aus (10) und (9)

$$
\frac{a^{2}+q}{q^{\prime}}=2^{2 i} \cdot 5^{2 k} \cdot q^{\prime}+2^{s} \cdot 5^{t} \cdot q^{\prime \prime}=2^{n+1+s-i} \cdot 5^{n+1+t-k} \cdot \frac{q^{\prime \prime}}{q^{\prime}}
$$

mit $q^{\prime} \mid q^{\prime \prime}$ und

$$
\frac{a^{2}+q}{q^{\prime 2}}=2^{2 i} \cdot 5^{2 k}+2^{s} \cdot 5^{t} \cdot \frac{q^{\prime \prime}}{q^{\prime}}=2^{n+1+s-i} \cdot 5^{n+1+t-k} \cdot \frac{q^{\prime \prime}}{q^{\prime 2}}
$$

mit

$$
q^{\prime 2} \mid q^{\prime \prime}
$$

Mit (11) folgt

$$
q^{\prime \prime}=q^{\prime 2} \quad \text { und daher } q=2^{s} \cdot 5^{t} \cdot q^{\prime 3} \quad(\text { gemäß (7)). }
$$

Weiterhin ergibt sich nun aus (12)

$$
q^{\prime}=\frac{2^{n+1+s-i} \cdot 5^{n+1+t-k}-2^{2 i} \cdot 5^{2 k}}{2^{s} \cdot 5^{t}}=2^{n+1-i} \cdot 5^{n+1-k}-2^{2 i-s} \cdot 5^{2 k-t}
$$

mit den im Vergleich zu (7) und (8) eingeschränkten Exponentenbedingungen $0 \leq i, k \leq n+1,0 \leq s \leq 2 i, 0 \leq t \leq 2 k$.

Wegen $\left(10, q^{\prime}\right)=1$ reduziert sich (15) zunächst auf vier Darstellungsformen für $q^{\prime}$ :

$$
\begin{aligned}
& i=k=n+1, \quad 0 \leq s, t \leq 2 n+2: q_{1}^{\prime}=1-2^{2 n+2-s} \cdot 5^{2 n+2-t}, \\
& s=2 i, \quad t=2 k, \quad 0 \leq i, k \leq n+1: q_{2}^{\prime}=2^{n+1-i} \cdot 5^{n+1-k}-1, \\
& k=n+1, \quad s=2 i, \quad 0 \leq i \leq n+1, \quad 0 \leq t \leq 2 n+2: q_{3}^{\prime}=2^{n+1-i}-5^{2 n+2-t}, \\
& i=n+1, \quad t=2 k, \quad 0 \leq k \leq n+1, \quad 0 \leq s \leq 2 n+2: q_{4}^{\prime}=5^{n+1-k}-2^{2 n+2-s} .
\end{aligned}
$$

Dabei scheidet (16) von vornherein aus wegen $q_{1}^{\prime} \leq 0$. Die anderen $q^{\prime}$ sind auch noch hinsichtlich des Kriteriums $q<a$ zu untersuchen. Nach (14) und (8) ist $q<a$ äquivalent $\mathrm{zu}$

$$
q^{\prime 2}<2^{i-s} \cdot 5^{k-t}
$$

und die Wahl von $s$ und $t$ in (17) führt wiederum zu keinem $q^{\prime} \geq 1$. Im Fall (18) müsste $1 \leq q_{3}^{\prime 2}<\frac{5^{n+1-t}}{2^{i}} \leq 5^{n+1-t}$ mit $t \leq n$ gelten im Widerspruch zur Abschätzung $q_{3}^{\prime}=$ 
$2^{n+1-i}-5^{2 n+2-t} \leq 2^{n+1}-5^{n+2}<0$ für alle $n \in \mathbb{N}$. Entsprechende Überlegungen führen nur bei $q_{4}^{\prime}$ zu keinem Widerspruch. Nach (19) und (20) gilt es nun, $q_{4}^{\prime}$ zu finden mit

$$
1 \leq q_{4}^{\prime 2}<2^{n+1-s} \cdot 5^{-k}
$$

Hieraus folgt $5^{k}<2^{n+1-s}$ mit $s \leq n$ und mit (19) $2^{s} \cdot 5^{2 k}<2^{2 n+2-s}<5^{n+1-k}$ wegen $q_{4}^{\prime} \geq 1$. Daher gilt $2^{s}<5^{n+1-3 k}$ mit $k \leq \frac{n}{3}$, insgesamt also eine Verschärfung der Bedingungen für $s, k$ gegenüber (19). Für die weiteren Überlegungen genügen aber die Schranken $0 \leq s, k \leq n$. Setzt man gemäß (19)

$$
(1 \leq) q_{4}^{\prime}=u-v \quad \text { mit } u:=5^{n+1-k} \text { und } v:=2^{2 n+2-s}
$$

so erhält man nach Division von $q_{4}^{\prime 2}$ durch $u v$ infolge von (21) die obere Abschätzung

$$
\frac{u}{v}+\frac{v}{u}-2<\frac{1}{10^{n+1}}
$$

\subsection{Bestimmung von $u$ und $v$}

Wir betrachten zuerst den Fall $n \geq 1$ und nehmen zunächst an, dass

$$
u_{k}(n)>v_{s}(n) \wedge u_{k}(n-1)>v_{s}(n-1), \quad 0 \leq s, k \leq n,
$$

gilt, wobei $u_{k}(n-1)=5^{n-k}$ und $v_{s}(n-1)=2^{2 n-s}$ ist. Dafür ist $k$ auf $0 \leq k \leq n-1$ einzuschränken. Dann kann die linke Seite von (23) folgendermaßen nach unten abgeschätzt werden:

$$
\frac{5}{4} \cdot \frac{u_{k}(n-1)}{v_{s}(n-1)}+\frac{4}{5} \cdot \frac{v_{s}(n-1)}{u_{k}(n-1)}-2>\frac{5}{4}+\frac{4}{5}-2=\frac{1}{20}>\frac{1}{10^{n+1}} .
$$

Dazu beachte man, dass die Funktion $f(x)=x+\frac{1}{x}$ für $x>1$ streng monoton wächst und $x=\frac{5}{4} \cdot \frac{u}{v}$ ein Produkt aus zwei Faktoren ist, die beide 1 übersteigen. (24) lässt sich also mit (23) nicht vereinbaren. Es verbleibt jetzt noch der Fall

$$
u_{k}(n)>v_{s}(n) \wedge u_{k}(n-1)<v_{s}(n-1), \quad 0 \leq s, k \leq n .
$$

Äquivalent dazu ist die Ungleichungskette

$$
\frac{2^{2 n+2}}{5^{n+1}}<\frac{2^{s}}{5^{k}}<\frac{2^{2 n}}{5^{n}} .
$$

Mithilfe des Faktors $\frac{5^{3}}{2^{7}}=\frac{125}{128}$, der noch näher bei 1 liegt als $\frac{2^{2}}{5^{1}}=\frac{4}{5}$, lässt sich $\frac{2^{2 n}}{5^{n}}$ mehrfach passend verkleinern, womit konkrete $\frac{2^{s}}{5^{k}}$ unter der Bedingung (27) erzeugt werden können. Hierbei ist zu beachten, dass $\frac{4}{5}<\frac{5^{3 l}}{2^{7 l}}<1, l \in \mathbb{N}$, genau dann gilt, wenn $l$ im 
Intervall [1;9] liegt. Damit können $s$ und $k$ die folgenden Werte annehmen, womit eine weitere Einschränkung von $n$ verbunden ist:

$$
s=2 n-7 l, \quad k=n-3 l, \quad n \geq \frac{7}{2} l, \quad l=1, \ldots, 9 .
$$

Gibt es noch andere $s$ und $k$, die (27) erfüllen? Hierzu betrachten wir die zu (27) äquivalente Ungleichungskette

$$
2^{2 n+2-s}<5^{n+1-k}<2^{2 n-s} \cdot 5
$$

Wegen $0 \leq s \leq n$ ist $2^{2 n+2-s} \geq 8$ mit der Folge $k \leq n-1$ und rechts $s \leq 2 n-3$. Nun beginnt ein hübscher „Schaukel-Algorithmus“: Man erhält weiter $2^{2 n+2-s} \geq 32$ und $k \leq n-2$, so dass rechts $s \leq 2 n-5$ folgt. Dann ist aber $2^{2 n+2-s} \geq 128, k \leq n-3$ und rechts $s \leq 2 n-7$. Damit ergibt sich aus (29)

$$
512 \cdot 2^{2 n-7-s}<625 \cdot 5^{n-3-k}<128 \cdot 2^{2 n-7-s} \cdot 5
$$

mit ausschließlich nicht-negativen Exponenten, wobei aber jetzt die Schranken von $k$ und $s$ stagnieren. Das Verfahren bricht an dieser Stelle ab. Aus (27) bzw. (29) folgt also einerseits die Exponentenbedingung

$$
s \leq 2 n-7 \quad \text { und } \quad k \leq n-3
$$

andererseits erweist sich der Fall $l=1$ in (28) wieder als eine Lösung von (27), wie man an der gültigen Ungleichungskette $512<625<128 \cdot 5$ in (30) erkennt. $s=2 n-7$ und $k=n-3$ sind also die maximalen Exponenten, für die die Ungleichungskette (27) erfüllt ist. $s$ und $k$ mit $l=2$ in (28) müssen nun die zweithöchsten Exponenten sein, denn angenommen, (27) würde durch ein Paar $(s, k)$ mit $2 n-14<s<2 n-7, n-6<k<$ $n-3$ erfüllt, dann ergäbe sich wegen $\frac{2^{s}}{5^{k}} \cdot \frac{2^{7}}{5^{3}}<\frac{2^{2 n}}{5^{n}}$ auch noch für $(s+7, k+3)$ mit $2 n-7<s+7<2 n, n-3<k+3<n$ eine Lösung. Diese Exponentenbedingungen widersprechen aber (31).

Da sich diese Überlegung für alle $l=3, \ldots, 9$ fortsetzen lässt, sind mit (28) tatsächlich alle Lösungen von (27) erfasst. Nach (22) folgt nun

$$
\frac{u_{k}(n)}{v_{s}(n)}+\frac{v_{s}(n)}{u_{k}(n)}-2=\frac{5}{4}\left(\frac{125}{128}\right)^{l}+\frac{4}{5}\left(\frac{128}{125}\right)^{l}-2 .
$$

Die Funktion auf der rechten Seite fällt offenbar streng monoton mit $l$. Das Minimum wird also für $l=9$ angenommen und ist größer als $9 \cdot 10^{-5}$. Andererseits lässt sich die rechte Seite von (23) mit

$$
\frac{1}{10^{n+1}} \leq \frac{1}{10^{\frac{7}{2}+l}}
$$

nach oben abschätzen, wobei das Maximum für $l=1$ angenommen wird und kleiner als $4 \cdot 10^{-5}$ ist. Damit gilt auch hier $\frac{u_{k}(n)}{v_{s}(n)}+\frac{v_{s}(n)}{u_{k}(n)}-2>\frac{1}{10^{n+1}}$ im Widerspruch zu (23). 
Unser Problem ist also auf $n=0$ reduziert. Es folgt $s=k=0, u_{0}(0)=5, v_{0}(0)=4$, $\frac{u_{0}(n)}{\nu_{0}(n)}+\frac{\nu_{0}(n)}{u_{0}(n)}-2=\frac{1}{20}<\frac{1}{10^{1}}$ gemäß (23), $q_{4}^{\prime}=1$ mit $i=1, t=0$ nach (19), $q=1$ nach (14), $a=2$ nach (8), $z=a_{0}=5$ nach (4) und (1). Damit gilt

Satz 1. 2,5 ist der einzige Dezimalbruch, der die Eigenschaft (1) besitzt.

\section{$2 g$-adische Systembrüche}

Im Weiteren wollen wir nun allgemein $g$-adische Systembrüche $a, a_{n} a_{n-1} \ldots a_{1} a_{0}$, $a \in \mathbb{N}^{*}, 0 \leq a_{v} \leq g-1(0 \leq v \leq n)$ mit der Eigenschaft

$$
\left(a, a_{n} a_{n-1} \ldots a_{1} a_{0}\right)_{g}=\frac{z}{a}, \quad z=\sum_{\nu=0}^{n} a_{\nu} \cdot g^{\nu}<g^{n+1},
$$

betrachten.

\section{1 $g=p^{\alpha}, p$ Primzahl, $\alpha \geq 1$}

Die Schritte (2)-(15) für $g=10$ lassen sich weitgehend analog auf $g=p^{\alpha}$ übertragen: Mit den alten Bezeichnungen erhält man jetzt $q^{\prime \prime}=q^{\prime 2}, q=p^{s} \cdot q^{\prime 3}$ und schließlich

$$
q^{\prime}=p^{\alpha(n+1)-i}-p^{2 i-s}
$$

mit den Exponentenbedingungen $1 \leq i \leq \alpha(n+1), 0 \leq s \leq 2 i$.

Wegen $\left(p, q^{\prime}\right)=1$ reduziert sich $\left(15^{\prime}\right)$ nun auf nur zwei Darstellungsformen für $q^{\prime}$ :

$$
\begin{aligned}
& 1 \leq i=\alpha(n+1), \quad 0 \leq s \leq 2 \alpha(n+1): q_{1}^{\prime}=1-p^{2 \alpha(n+1)-s}, \\
& s=2 i, \quad 1 \leq i \leq \alpha(n+1): q_{2}^{\prime}=p^{\alpha(n+1)-i}-1 .
\end{aligned}
$$

Einerseits gilt $q_{1}^{\prime} \leq 0$, andererseits ist $q<a$ wegen $a=p^{i} \cdot q^{\prime}$ jetzt äquivalent zu $\left(20^{\prime}\right)$ $q^{\prime 2}<p^{i-s}$, so dass für $q^{\prime}=q_{2}^{\prime}$ mit $s=2 i$ folgt: $q_{2}^{\prime 2}<p^{-i} \leq p^{-1}-$ im Widerspruch zu $q_{2}^{\prime} \geq 1$. Daher gilt

Satz 2. Ist $g>1$ eine Primzahlpotenz, so gibt es im zugehörigen g-adischen System keine Darstellung gemäß $\left(1^{\prime}\right)$.

\section{2 $g$-adische Systeme mit mindestens einer Darstellung ${ }^{2}$}

Ersetzt man 10 durch $g$, so erhält man

$$
\frac{z}{g^{n+1}}=\frac{q}{a}
$$

\footnotetext{
${ }^{2}$ Unter einer „Darstellung“ sei hier und im Folgenden die Darstellung gemäß $\left(1^{\prime}\right)$ gemeint.
} 
mit

$$
z=a^{2}+q, \quad q=\frac{a^{3}}{g^{n+1}-a}<a .
$$

Daraus folgt unmittelbar

Satz 3. Ein g-adisches System hat genau dann mindestens eine Darstellung, wenn ein $n \in \mathbb{N}$ und ein natürliches $q, 1 \leq q<a$, $q \mid a^{3}$, existieren mit $g^{n+1}=\frac{a^{3}}{q}+a$. Die zugehörige Darstellung ist

$$
\left(a, a_{n} a_{n-1} \ldots a_{1} a_{0}\right)_{g}=\frac{a^{2}+q}{a}, \quad a^{2}+q=\sum_{\nu=0}^{n} a_{\nu} \cdot g^{\nu} .
$$

Beispiele. $n=0$ (Darstellung bezogen auf 1 Nachkommastelle):

$q=1, a=2: g=10,(2,5)_{10}=\frac{5}{2}$

$q=4, a=6: g=60,(6,[40])_{60}=\left(\frac{[40]}{6}\right)_{60}\left(\text { gemeinsamer Wert: } 6 \frac{2}{3}\right)^{3}$

$n=1$ (Darstellung bezogen auf 2 Nachkommastellen):

$q=2, a=4: g=6,(4,30)_{6}=\left(\frac{30}{4}\right)_{6}\left(\right.$ gemeinsamer Wert: $\left.4 \frac{1}{2}\right)$

$q=27, a=36: g=42,([36],[31][21])_{42}=\left(\frac{[31][21]}{[36]}\right)_{42}\left(\right.$ gemeinsamer Wert: $\left.36 \frac{3}{4}\right)$

Bemerkung. Die Frage, ob es Darstellungen gibt, die sich auf mehr als 2 Nachkommastellen beziehen $(n \geq 2)$, ist offen.

Wir wollen nun die folgende Tabelle studieren, in der alle Werte $g$ unterhalb von 10000 mit $g=\frac{a^{3}}{q}+a$ unter der Bedingung $1 \leq q<a, q \mid a^{3}(n=0)$ der Größe nach aufgelistet

\begin{tabular}{|c|c|c|c|c|c|c|}
\hline $\begin{array}{c}\mathbf{1 0} \\
2 * 1\end{array}$ & $\begin{array}{c}30 \\
3 * 1\end{array}$ & $\begin{array}{c}\mathbf{3 6} \\
4 * 2=6^{2}\end{array}$ & $\begin{array}{c}\mathbf{6 0} \\
6 * 4\end{array}$ & $\begin{array}{c}\mathbf{6 8} \\
4 * 1\end{array}$ & $\begin{array}{c}\mathbf{7 8} \\
6 * 3\end{array}$ & \\
\hline $\begin{array}{l}114 \\
6 * 2\end{array}$ & $\begin{array}{l}130 \\
5 * 1\end{array}$ & $\begin{array}{c}135 \\
10 * 8\end{array}$ & $\begin{array}{l}\mathbf{1 3 6} \\
8 * 4\end{array}$ & & & \\
\hline $\begin{array}{c}\mathbf{2 0 4} \\
12 * 9\end{array}$ & $\begin{array}{c}\mathbf{2 1 0} \\
10 * 5\end{array}$ & $\begin{array}{l}222 \\
6 * 1\end{array}$ & $\begin{array}{c}\mathbf{2 2 8} \\
12 * 8\end{array}$ & $\begin{array}{l}\mathbf{2 5 2} \\
9 * 3\end{array}$ & $\begin{array}{c}\mathbf{2 6 0} \\
10 * 4\end{array}$ & $\begin{array}{l}\mathbf{2 6 4} \\
8 * 2\end{array}$ \\
\hline $\begin{array}{c}300 \\
12 * 6\end{array}$ & $\begin{array}{l}350 \\
7 * 1\end{array}$ & $\begin{array}{c}357 \\
14 * 8\end{array}$ & $\begin{array}{c}390 \\
15 * 9\end{array}$ & & & \\
\hline $\begin{array}{c}406 \\
14 * 7\end{array}$ & $\begin{array}{c}\mathbf{4 4 4} \\
12 * 4\end{array}$ & & & & & \\
\hline $\begin{array}{c}\mathbf{5 0 4} \\
18 * 12\end{array}$ & $\begin{array}{c}\mathbf{5 1 0} \\
10 * 2\end{array}$ & $\begin{array}{c}\mathbf{5 2 0} \\
8 * 1=20 * 16\end{array}$ & $\begin{array}{c}\mathbf{5 2 8} \\
16 * 8\end{array}$ & $\begin{array}{c}\mathbf{5 8 8} \\
12 * 3\end{array}$ & & \\
\hline $\begin{array}{c}666 \\
18 * 9\end{array}$ & $\begin{array}{c}\mathbf{6 9 0} \\
15 * 5\end{array}$ & & & & & \\
\hline
\end{tabular}
sind, und zur weiteren Theoriebildung beitragen.

\footnotetext{
${ }^{3}$ Einziffrige Zahlen werden durch eckige Klammern gekennzeichnet, um Missverständnisse zu vermeiden.
} 


\begin{tabular}{|c|c|c|c|c|c|c|c|}
\hline $\begin{array}{c}700 \\
14 * 4\end{array}$ & $\begin{array}{l}738 \\
9 * 1\end{array}$ & $\begin{array}{c}747 \\
18 * 8\end{array}$ & $\begin{array}{c}792 \\
24 * 18\end{array}$ & & & & \\
\hline $\begin{array}{c}\mathbf{8 2 0} \\
20 * 10\end{array}$ & $\begin{array}{c}876 \\
12 * 2 \\
\end{array}$ & $\begin{array}{c}\mathbf{8 8 8} \\
24 * 16\end{array}$ & & & & & \\
\hline \multicolumn{8}{|c|}{$\begin{array}{c}990 \\
18 * 6=22 * 11 \\
\end{array}$} \\
\hline $\begin{array}{l}1010 \\
10 * 1\end{array}$ & $\begin{array}{l}1020 \\
20 * 8\end{array}$ & $\begin{array}{c}1030 \\
30 * 27\end{array}$ & $\begin{array}{l}1040 \\
16 * 4\end{array}$ & $\begin{array}{l}1050 \\
21 * 9\end{array}$ & & & \\
\hline $\begin{array}{c}1110 \\
30 * 25\end{array}$ & $\begin{array}{l}1140 \\
15 * 3\end{array}$ & $\begin{array}{c}\mathbf{1 1 5 5} \\
30 * 24\end{array}$ & $\begin{array}{c}\mathbf{1 1 7 6} \\
24 * 12\end{array}$ & & & & \\
\hline $\begin{array}{l}1342 \\
11 * 1 \\
\end{array}$ & $\begin{array}{l}1344 \\
21 * 7 \\
\end{array}$ & $\begin{array}{l}1353 \\
22 * 8 \\
\end{array}$ & $\begin{array}{c}\mathbf{1 3 6 4} \\
33 * 27 \\
\end{array}$ & \begin{tabular}{|c|}
$\mathbf{1 3 7 8}$ \\
$26 * 13$ \\
\end{tabular} & $\begin{array}{c}1380 \\
30 * 20 \\
\end{array}$ & $\begin{array}{l}1386 \\
14 * 2 \\
\end{array}$ & \\
\hline $\begin{array}{c}1400 \\
28 * 16\end{array}$ & $\begin{array}{l}1476 \\
18 * 4\end{array}$ & $\begin{array}{c}1494 \\
36 * 32\end{array}$ & $\begin{array}{c}\mathbf{1 5 3 0} \\
30 * 18 \\
\end{array}$ & $\begin{array}{l}\mathbf{1 5 6 0} \\
24 * 9 \\
\end{array}$ & $\begin{array}{c}\mathbf{1 5 9 6} \\
28 * 14\end{array}$ & $\begin{array}{l}1620 \\
20 * 5\end{array}$ & \\
\hline $\begin{array}{l}1740 \\
12 * 1 \\
\end{array}$ & $\begin{array}{c}1750 \\
35 * 25\end{array}$ & $\begin{array}{l}1752 \\
24 * 8\end{array}$ & $\begin{array}{c}1764 \\
36 * 27=42^{2}\end{array}$ & $\begin{array}{c}\mathbf{1 8 3 0} \\
30 * 15 \\
\end{array}$ & $\begin{array}{l}1962 \\
18 * 3 \\
\end{array}$ & $\begin{array}{c}1980 \\
36 * 24 \\
\end{array}$ & \\
\hline $\begin{array}{l}2020 \\
20 * 4 \\
\end{array}$ & $\begin{array}{c}2040 \\
40 * 32 \\
\end{array}$ & $\begin{array}{l}\mathbf{2 0 6 4} \\
16 * 2 \\
\end{array}$ & $\begin{array}{c}\mathbf{2 0 8 0} \\
32 * 16 \\
\end{array}$ & $\begin{array}{c}\mathbf{2 1 0 0} \\
42 * 36 \\
\end{array}$ & & & \\
\hline $\begin{array}{l}2210 \\
13 * 1\end{array}$ & $\begin{array}{l}2214 \\
27 * 9\end{array}$ & $\begin{array}{l}2223 \\
26 * 8\end{array}$ & $\begin{array}{c}\mathbf{2 2 3 6} \\
39 * 27 \\
\end{array}$ & $\begin{array}{c}\mathbf{2 2 8 0} \\
30 * 12 \\
\end{array}$ & $\begin{array}{l}2328 \\
24 * 6\end{array}$ & $\begin{array}{c}\mathbf{2 3 4 6} \\
34 * 17 \\
\end{array}$ & \\
\hline $\begin{array}{c}2600 \\
40 * 25\end{array}$ & $\begin{array}{c}\mathbf{2 6 2 8} \\
36 * 18\end{array}$ & $\begin{array}{l}\mathbf{2 6 8 4} \\
22 * 4 \\
\end{array}$ & $\begin{array}{c}\mathbf{2 6 8 8} \\
42 * 28 \\
\end{array}$ & & & & \\
\hline $\begin{array}{c}\mathbf{2 7 0 6} \\
44 * 32\end{array}$ & $\begin{array}{c}\mathbf{2 7 3 0} \\
30 * 10\end{array}$ & $\begin{array}{l}\mathbf{2 7 5 8} \\
14 * 1\end{array}$ & $\begin{array}{l}2772 \\
28 * 8\end{array}$ & $\begin{array}{c}\mathbf{2 7 8 6} \\
42 * 27\end{array}$ & $\begin{array}{c}\mathbf{2 9 2 6} \\
38 * 19\end{array}$ & $\begin{array}{l}2934 \\
18 * 2\end{array}$ & $\begin{array}{c}\mathbf{2 9 5 2} \\
36 * 16\end{array}$ \\
\hline $\begin{array}{l}\mathbf{3 0 3 0} \\
30 * 9\end{array}$ & $\begin{array}{l}\mathbf{3 1 0 8} \\
21 * 3\end{array}$ & $\begin{array}{c}\mathbf{3 1 2 0} \\
48 * 36\end{array}$ & $\begin{array}{c}3129 \\
42 * 24 \\
\end{array}$ & $\begin{array}{l}3150 \\
25 * 5\end{array}$ & $\begin{array}{l}3164 \\
28 * 7\end{array}$ & $\begin{array}{c}3175 \\
50 * 40 \\
\end{array}$ & $\begin{array}{c}3240 \\
40 * 20\end{array}$ \\
\hline $\begin{array}{c}3300 \\
33 * 11 \\
\end{array}$ & $\begin{array}{l}3390 \\
15 * 1 \\
\end{array}$ & $\begin{array}{l}3405 \\
30 * 8 \\
\end{array}$ & $\begin{array}{c}3420 \\
45 * 27 \\
\end{array}$ & $\begin{array}{l}3480 \\
24 * 4 \\
\end{array}$ & $\begin{array}{c}3504 \\
48 * 32 \\
\end{array}$ & $\begin{array}{c}3570 \\
42 * 21 \\
\end{array}$ & \\
\hline $\begin{array}{c}\mathbf{3 6 4 0} \\
56 * 49\end{array}$ & $\begin{array}{c}3690 \\
45 * 25\end{array}$ & $\begin{array}{c}3916 \\
44 * 22\end{array}$ & $\begin{array}{c}3924 \\
36 * 12\end{array}$ & $\begin{array}{l}\mathbf{4 0 2 0} \\
20 * 2\end{array}$ & $\begin{array}{l}\mathbf{4 0 2 6} \\
33 * 9\end{array}$ & $\begin{array}{c}\mathbf{4 0 4 0} \\
40 * 16\end{array}$ & $\begin{array}{c}4060 \\
60 * 54\end{array}$ \\
\hline $\begin{array}{l}4112 \\
16 * 1 \\
\end{array}$ & $\begin{array}{l}\mathbf{4 1 2 8} \\
32 * 8 \\
\end{array}$ & $\begin{array}{c}\mathbf{4 1 4 4} \\
48 * 27 \\
\end{array}$ & $\begin{array}{c}\mathbf{4 1 5 8} \\
42 * 18 \\
\end{array}$ & $\begin{array}{r}4278 \\
46 * 23 \\
\end{array}$ & $\begin{array}{c}4380 \\
60 * 50 \\
\end{array}$ & & \\
\hline $\begin{array}{l}4420 \\
26 * 4 \\
\end{array}$ & $\begin{array}{c}4428 \\
54 * 36\end{array}$ & $\begin{array}{c}4446 \\
52 * 32 \\
\end{array}$ & $\begin{array}{l}\mathbf{4 5 3 0} \\
30 * 6 \\
\end{array}$ & $\begin{array}{c}4560 \\
60 * 48 \\
\end{array}$ & $\begin{array}{c}\mathbf{4 6 0 2} \\
39 * 13 \\
\end{array}$ & $\begin{array}{l}\mathbf{4 6 3 2} \\
24 * 3 \\
\end{array}$ & $\begin{array}{c}\mathbf{4 6 5 6} \\
48 * 24 \\
\end{array}$ \\
\hline $\begin{array}{c}4860 \\
60 * 45 \\
\end{array}$ & $\begin{array}{l}4930 \\
17 * 1 \\
\end{array}$ & $\begin{array}{l}4947 \\
34 * 8 \\
\end{array}$ & $\begin{array}{c}4964 \\
51 * 27 \\
\end{array}$ & $\begin{array}{c}4981 \\
68 * 64 \\
\end{array}$ & $\begin{array}{c}\mathbf{5 0 5 0} \\
50 * 25 \\
\end{array}$ & $\begin{array}{c}\mathbf{5 1 6 6} \\
63 * 49 \\
\end{array}$ & $\begin{array}{l}\mathbf{5 2 2 0} \\
36 * 9 \\
\end{array}$ \\
\hline $\begin{array}{c}\mathbf{5 3 3 4} \\
42 * 14\end{array}$ & $\begin{array}{l}\mathbf{5 3 4 6} \\
22 * 2 \\
\end{array}$ & $\begin{array}{c}\mathbf{5 3 6 8} \\
44 * 16\end{array}$ & $\begin{array}{c}\mathbf{5 3 9 0} \\
66 * 54 \\
\end{array}$ & $\begin{array}{l}\mathbf{5 4 3 0} \\
30 * 5 \\
\end{array}$ & $\begin{array}{c}\mathbf{5 4 6 0} \\
52 * 26=60 * 40\end{array}$ & $\begin{array}{l}\mathbf{5 5 1 6} \\
28 * 4 \\
\end{array}$ & $\begin{array}{c}\mathbf{5 5 4 4} \\
56 * 32 \\
\end{array}$ \\
\hline $\begin{array}{l}\mathbf{5 8 5 0} \\
18 * 1 \\
\end{array}$ & $\begin{array}{l}\mathbf{5 8 6 8} \\
36 * 8 \\
\end{array}$ & $\begin{array}{c}\mathbf{5 8 8 6} \\
54 * 27 \\
\end{array}$ & $\begin{array}{c}\mathbf{5 9 0 4} \\
72 * 64 \\
\end{array}$ & $\begin{array}{c}\mathbf{6 0 6 0} \\
60 * 36 \\
\end{array}$ & & & \\
\hline $\begin{array}{c}\mathbf{6 1 2 0} \\
45 * 15 \\
\end{array}$ & $\begin{array}{l}\mathbf{6 1 6 0} \\
35 * 7 \\
\end{array}$ & $\begin{array}{c}6192 \\
48 * 18 \\
\end{array}$ & $\begin{array}{c}\mathbf{6 1 9 5} \\
70 * 56 \\
\end{array}$ & $\begin{array}{c}\mathbf{6 2 1 6} \\
42 * 12 \\
\end{array}$ & $\begin{array}{c}\mathbf{6 3 0 0} \\
50 * 20 \\
\end{array}$ & $\begin{array}{c}\mathbf{6 3 2 8} \\
56 * 28 \\
\end{array}$ & $\begin{array}{c}\mathbf{6 4 4 0} \\
40 * 10 \\
\end{array}$ \\
\hline $\begin{array}{l}\mathbf{6 5 8 8} \\
27 * 3 \\
\end{array}$ & $\begin{array}{c}\mathbf{6 6 0 0} \\
66 * 44\end{array}$ & $\begin{array}{c}\mathbf{6 6 1 5} \\
54 * 24\end{array}$ & $\begin{array}{l}6630 \\
39 * 9\end{array}$ & $\begin{array}{c}6669 \\
78 * 72 \\
\end{array}$ & $\begin{array}{c}\mathbf{6 7 1 0} \\
55 * 25 \\
\end{array}$ & $\begin{array}{l}\mathbf{6 7 8 0} \\
30 * 4 \\
\end{array}$ & $\begin{array}{c}\mathbf{6 7 8 6} \\
58 * 29 \\
\end{array}$ \\
\hline $\begin{array}{c}\mathbf{6 8 1 0} \\
60 * 32 \\
\end{array}$ & $\begin{array}{l}\mathbf{6 8 7 8} \\
19 * 1 \\
\end{array}$ & $\begin{array}{l}\mathbf{6 8 9 7} \\
38 * 8 \\
\end{array}$ & & & & & \\
\hline $\begin{array}{c}\mathbf{6 9 1 6} \\
57 * 27\end{array}$ & $\begin{array}{c}\mathbf{6 9 3 0} \\
70 * 50 \\
\end{array}$ & $\begin{array}{c}\mathbf{6 9 3 5} \\
76 * 64 \\
\end{array}$ & $\begin{array}{l}\mathbf{6 9 3 6} \\
24 * 2 \\
\end{array}$ & $\begin{array}{c}\mathbf{6 9 6 0} \\
48 * 16 \\
\end{array}$ & $\begin{array}{c}\mathbf{6 9 8 4} \\
72 * 54 \\
\end{array}$ & $\begin{array}{c}\mathbf{7 0 7 0} \\
70 * 49 \\
\end{array}$ & $\begin{array}{c}\mathbf{7 2 6 0} \\
60 * 30 \\
\end{array}$ \\
\hline $\begin{array}{c}7750 \\
62 * 31 \\
\end{array}$ & $\begin{array}{c}\mathbf{7 7 8 8} \\
44 * 11\end{array}$ & $\begin{array}{l}\mathbf{7 8 1 2} \\
36 * 6\end{array}$ & $\begin{array}{c}\mathbf{7 8 4 8} \\
72 * 48 \\
\end{array}$ & \begin{tabular}{|c|}
$\mathbf{7 8 5 4}$ \\
$51 * 17$ \\
\end{tabular} & & & \\
\hline
\end{tabular}




\begin{tabular}{|c|c|c|c|c|c|c|c|}
\hline $\mathbf{8 0 2 0}$ & $\mathbf{8 0 4 0}$ & $\mathbf{8 0 5 2}$ & $\mathbf{8 0 6 0}$ & $\mathbf{8 0 8 0}$ & $\mathbf{8 2 2 4}$ & $\mathbf{8 2 5 6}$ & $\mathbf{8 2 7 4}$ \\
$20 * 1$ & $40 * 8$ & $66 * 36$ & $60 * 27$ & $80 * 64$ & $32 * 4$ & $64 * 32$ & $42 * 9$ \\
\hline $\mathbf{8 3 1 6}$ & $\mathbf{8 6 1 0}$ & $\mathbf{8 6 4 5}$ & $\mathbf{8 7 0 0}$ & $\mathbf{8 7 7 8}$ & & & \\
$84 * 72$ & $35 * 5$ & $70 * 40$ & $60 * 25$ & $66 * 33$ & & & \\
\hline $\mathbf{8 8 0 2}$ & $\mathbf{8 8 1 4}$ & $\mathbf{8 8 4 0}$ & $\mathbf{8 8 6 6}$ & $\mathbf{9 0 3 0}$ & $\mathbf{9 0 6 0}$ & $\mathbf{9 0 9 0}$ & \\
$54 * 18$ & $26 * 2$ & $52 * 16$ & $78 * 54$ & $30 * 3$ & $60 * 24$ & $90 * 81$ & \\
\hline $\mathbf{9 2 0 4}$ & $\mathbf{9 2 6 4}$ & $\mathbf{9 2 8 2}$ & $\mathbf{9 3 0 3}$ & $\mathbf{9 3 1 6}$ & $\mathbf{9 3 2 4}$ & $\mathbf{9 3 4 5}$ & $\mathbf{9 3 9 4}$ \\
$78 * 52$ & $48 * 12$ & $21 * 1$ & $42 * 8$ & $68 * 34$ & $63 * 27$ & $84 * 64$ & $77 * 49$ \\
\hline $\mathbf{9 4 5 0}$ & $\mathbf{9 4 9 2}$ & $\mathbf{9 8 0 4}$ & $\mathbf{9 8 1 0}$ & $\mathbf{9 8 6 0}$ & $\mathbf{9 8 7 0}$ & $\mathbf{9 8 9 4}$ & \\
$75 * 45$ & $84 * 63$ & $57 * 19$ & $90 * 75$ & $34 * 4$ & $70 * 35$ & $68 * 32$ & \\
\hline
\end{tabular}

Tabelle 1 für $2 \leq g \leq 10000, g=a * q:=\frac{a^{3}}{q}+a, 1 \leq q<a, q \mid a^{3}$

Nach Satz 2 treten für $g$ keine Primzahlpotenzen auf, so dass $g$ mindestens zwei verschiedene Primfaktoren enthält. Speziell gibt es Zahlen $g$, die sich in genau zwei verschiedene Primfaktoren zerlegen lassen, z.B. 10 und $4981(=17 \cdot 293)$.

Es kommen gerade und ungerade $g$ vor, wobei alle Endziffern ausgeschöpft werden, aber die geraden $g$ dominieren (in der Tabelle) bei Weitem. Ungerades $g$ zieht offenbar gerades $a$ nach sich. Darüber hinaus erhält man sofort folgendes Kriterium:

$$
\begin{aligned}
& g \text { ist ungerade genau dann, wenn gilt: } \\
& a=2^{i} a^{\prime}, q=2^{3 i} q^{\prime}, i \geq 1, a^{\prime}, q^{\prime} \text { ungerade. }
\end{aligned}
$$

Dabei gilt $q^{\prime} \mid a^{\prime 3}$. Weiterhin hat $g$ die Produktdarstellungen

$$
g=\frac{a}{(a, q)} \cdot\left(\frac{a^{2}}{\frac{q}{(a, q)}}+(a, q)\right)=\frac{a}{(a, q)} \cdot\left(\frac{(a, q)^{2}}{\frac{q}{(a, q)}} \cdot\left(\frac{a}{(a, q)}\right)^{2}+(a, q)\right) .
$$

In diesem Zusammenhang bietet sich auch an, die Werte $q$ zu klassifizieren:

$$
\begin{gathered}
(q<a) \wedge(q \mid a), \\
(q<a) \wedge(q \nmid a) \wedge\left(q \mid a^{2}\right), \\
(q<a) \wedge\left(q \nmid a^{2}\right) \wedge\left(q \mid a^{3}\right) .
\end{gathered}
$$

In den Fällen (36) und (36') lässt sich (35) vereinfachen, und man erhält $g=\frac{a}{q} \cdot\left(a^{2}+q\right)$ bzw. $g=\frac{a}{(a, q)} \cdot(a, q) \cdot\left(\frac{a^{2}}{q}+1\right)$, wobei kein Faktor gleich 1 ist. Man sieht direkt - auch ohne Rückgriff auf das Kriterium (34) - leicht ein, dass in diesen Fällen $g$ stets gerade ist.

\section{3 $g$-adische Systeme mit mindestens zwei Darstellungen}

In der Tabelle 1 fallen weiterhin die Werte $g=520,990,5460$ auf, die nach Satz 3 (Fall $n=0$ ) jeweils mindestens zwei verschiedene auf die 1 . Nachkommastelle bezogene Dar- 
stellungen besitzen, und zwar

bzw.

$$
\begin{aligned}
(8,[65])_{520} & =\left(\frac{[65]}{8}\right)_{520} & & \left(\text { gemeinsamer Wert: } 8 \frac{1}{8}\right), \\
([20],[416])_{520} & =\left(\frac{[416]}{[20]}\right)_{520} & & \left(\text { gemeinsamer Wert: } 20 \frac{4}{5}\right)
\end{aligned}
$$

$$
\begin{array}{ll}
([18],[330])_{990}=\left(\frac{[330]}{[18]}\right)_{990} & \left(\text { gemeinsamer Wert: } 18 \frac{1}{3}\right), \\
([22],[495])_{990}=\left(\frac{[495]}{[22]}\right)_{990} & \left(\text { gemeinsamer Wert: } 22 \frac{1}{2}\right)
\end{array}
$$

bzw.

$$
\begin{array}{ll}
([52],[2730])_{5460}=\left(\frac{[2730]}{[52]}\right)_{5460} & \left(\text { gemeinsamer Wert: } 52 \frac{1}{2}\right), \\
([60],[3640])_{5460}=\left(\frac{[3640]}{[60]}\right)_{5460} & \left(\text { gemeinsamer Wert: } 60 \frac{2}{3}\right)
\end{array}
$$

und möglicherweise noch weitere, falls für diese $g$ außerdem noch die Identität $g^{n+1}=$ $\frac{b^{3}}{r}+b\left(r<b, r \mid b^{3}\right)$ für ein $n \geq 1$ gilt. (Im Bereich der Tabelle 1 ist dies nicht der Fall.) Es stellt sich nun natürlicherweise die Frage, ob es unendlich viele $g$ mit (mindestens) zwei Darstellungen gibt und ob man gegebenenfalls zumindest eine unendliche Teilmenge dieser $g$ konkret angeben kann.

Dabei sollen zwei Darstellungen bezüglich $g$, die sich jeweils auf die 1. Nachkommastelle beziehen, als 1/1-Darstellungen von $g$ bezeichnet werden. Außerdem gehen wir noch kurz auf 1/2-Darstellungen von $g$ ein, d.h. auf Darstellungen bezüglich $g$, von denen sich die eine auf die 1. Nachkommastelle, die andere auf die ersten beiden Nachkommastellen bezieht.

\subsection{1 $g$-adische Systeme mit 1/1-Darstellungen}

Hier sind $g$ zu finden mit

$$
g=\frac{a^{3}}{q}+a=\frac{b^{3}}{r}+b, \quad a \neq b, \quad q<a, \quad q\left|a^{3}, \quad r<b, \quad r\right| b^{3} .
$$

Sei o.B.d.A. $a<b$. Die Tabelle 1 lieferte die Beispiele $g=520, g=990$ und $g=5460$. Wir wollen nur den speziellen Fall $q|a, r| b$ betrachten, wie er für $g=990$ vorliegt, und erhalten mit $a=\lambda q, b=\mu r, 1<\lambda \leq a, 1<\mu \leq b$

$$
g=a(\lambda a+1)=b(\mu b+1), \quad 2 \leq \mu<\lambda,
$$

bzw.

$$
\frac{a}{b}=\frac{\mu b+1}{\lambda a+1}, \quad \mu|b, \quad \lambda| a, \quad 2 \leq \mu<\lambda .
$$


Mit $s=(a, b)$ schreiben wir $a=\alpha s, b=\beta s$ :

$$
\frac{\alpha s}{\beta s}=\frac{\mu \beta s+1}{\lambda \alpha s+1}, \quad \alpha<\beta, \quad(\alpha, \beta)=1, \quad \mu|\beta s, \quad \lambda| \alpha s, \quad 2 \leq \mu<\lambda .
$$

Für die Zahl $k$, mit der der Bruch $\frac{\alpha}{\beta}$ zu $\frac{\mu \beta s+1}{\lambda \alpha s+1}$ erweitert wird, gilt $k=\frac{\lambda \alpha s-\mu \beta s}{\beta-\alpha}$, woraus einerseits $k+\lambda s=\frac{(\lambda-\mu) \beta s}{\beta-\alpha}$, andererseits $k+\mu s=\frac{(\lambda-\mu) \alpha s}{\beta-\alpha}$ folgt. Somit ergibt sich

$$
\beta-\alpha \mid(\lambda-\mu) s .
$$

Wir nehmen nun eine erneute Spezialisierung vor:

$$
\beta-\alpha=(\lambda-\mu) s \text {. }
$$

Dann ist

$$
k+\lambda s=\beta, \quad k+\mu s=\alpha,
$$

und wegen der Erweiterungseigenschaft von $k$ ergibt sich aus $\left(38^{\prime}\right)$ :

$$
k(k+\mu s)=\mu(k+\lambda s) s+1, \quad k(k+\lambda s)=\lambda(k+\mu s) s+1 .
$$

Aus beiden Gleichungen erhält man

$$
k^{2}-1=\mu \lambda s^{2},
$$

was nach (40) zu $k^{2}-1=(\alpha-k)(\beta-k)$ mit $k=\frac{\alpha \beta+1}{\alpha+\beta}$ führt, woraus wieder wegen (40) die Darstellungen

$$
\mu s=\frac{\alpha^{2}-1}{\alpha+\beta}, \quad \lambda s=\frac{\beta^{2}-1}{\alpha+\beta},
$$

folgen. Einzellösungen des Systems (42) unter den Bedingungen aus (38') sind nun leicht zu finden. Wählt man z.B. $\mu=s=2$ und $\alpha$ ungerade, d.h. $\alpha=2 \gamma-1$, so ist nach (42) $\beta=\gamma^{2}-3 \gamma+1$ und $\lambda=\frac{1}{2}(\gamma-2)(\gamma-3)$. Dabei muss nach (38) $\gamma \geq 5$ sein. Es folgt

$$
\frac{(2 \gamma-1) \cdot 2}{\left(\gamma^{2}-3 \gamma+1\right) \cdot 2}=\frac{2\left(\gamma^{2}-3 \gamma+1\right) \cdot 2+1}{\frac{1}{2}(\gamma-2)(\gamma-3)(2 \gamma-1) \cdot 2+1} \text {. }
$$

Hier ist $\mu \mid \beta s$ trivialerweise allgemein erfüllt. $2=\mu<\lambda \wedge \lambda \mid \alpha s$ kann offenbar nur für endlich viele $\alpha$ erfüllt sein, hier für $\alpha_{1}=9, \beta_{1}=11$ mit $\lambda_{1}=3$ oder $\alpha_{2}=15, \beta_{2}=41$ mit $\lambda_{2}=15$. Damit erhält man die Darstellungen $\frac{9}{11}=\frac{18}{22}=\frac{2 \cdot 22+1}{3 \cdot 18+1}$ mit $q=6, r=11$, $k=5$ sowie $\frac{15}{41}=\frac{30}{82}=\frac{2 \cdot 82+1}{15 \cdot 30+1}$ mit $q=2, r=41, k=11$.

Nach Satz 3 führt die erste zu einem bereits bekannten Beispiel $(g=990)$, die zweite zu einem neuen:

$$
\begin{aligned}
& ([30],[902])_{13530}=\left(\frac{[902]}{[30]}\right)_{13530} \text { sowie }([82],[6765])_{13530}=\left(\frac{[6765]}{[82]}\right)_{13530} \\
& \left(\text { gemeinsame Werte: } 30 \frac{1}{15} \text { bzw. } 82 \frac{1}{2}\right) .
\end{aligned}
$$

Unser Ziel ist es aber, unendlich viele $\alpha, \beta$ zu finden, die $\left(38^{\prime}\right)$ erfüllen. Dazu bietet sich an, möglichst schwache Teilbarkeitsbedingungen zu fordern, d.h. $\mu=2$ und $\lambda=3 \mathrm{zu}$ wählen. 
Nach (41) folgt $k^{2}-1=6 s^{2}$ mit ungeradem $k$, etwa $k=2 m-1$, und daraus $2 m(m-1)=3 s^{2}$ mit geradem $s(s=2 \sigma)$. Damit ist die erste Bedingung $\mu \mid \beta s$ bereits erfüllt. Wir erhalten

$$
3 \sigma^{2}=\frac{1}{2} m(m-1) .
$$

Aus (40) folgt $m=\frac{1}{2}(\alpha-4 \sigma+1)=\gamma-2 \sigma$ mit $\alpha=2 \gamma-1$ (wegen ungeradem $k$ und $\mu=2)$, also

$$
3 \sigma^{2}=\frac{1}{2}(\gamma-2 \sigma)(\gamma-2 \sigma-1) .
$$

Wir zeigen zunächst, dass $m$ aus (43) bzw. $\gamma$ aus (43') stets ungerade ist.

Angenommen, es sei $m=2 t$ :

Im Fall $3 \mid 2 t-1$ existiert dann ein ungerades $l\left(l=2 l^{\prime}-1\right)$ mit $2 t-1=3 l$. Es folgt $t=3 l^{\prime}-1$ und $\sigma^{2}=\left(3 l^{\prime}-1\right)\left(2 l^{\prime}-1\right)$ mit teilerfremden quadratischen Faktoren. Mit den Bezeichnungen $3 l^{\prime}-1=c^{2}, 2 l^{\prime}-1=d^{2}$ folgt $2 c^{2}-1=3 d^{2}$ mit ungeradem $d$ $\left(d=2 d^{\prime}-1\right)$. Hieraus erhält man $c^{2}=2\left(3 d^{\prime}\left(d^{\prime}-1\right)+1\right)$ mit ungeradem zweiten Faktor, was zum Widerspruch führt.

Im Fall 3| $t(t=3 l)$ erhalten wir die Darstellung $\sigma^{2}=l(6 l-1)$, wiederum mit teilerfremden quadratischen Faktoren $\left(c^{2}, d^{2}\right)$. Es folgt $6 c^{2}-1=d^{2}$ mit ungeradem $d\left(d=2 d^{\prime}-1\right)$ und hieraus $3 c^{2}=2 d^{\prime}\left(d^{\prime}-1\right)+1$, wobei aber $d^{\prime}\left(d^{\prime}-1\right)$ nur die Reste 0 oder 2 mod 3 annehmen kann, was erneut zum Widerspruch führt.

$m$ ist also ungerade. Mit $m=2 t+1$ ergibt sich daher aus (43)

$$
3 \sigma^{2}=(2 t+1) t
$$

bzw. aus $\left(43^{\prime}\right)$ mit $\gamma=2 \delta+1$ :

$$
3 \sigma^{2}=(2 \delta-2 \sigma+1)(\delta-\sigma) .
$$

Die folgende Tabelle gibt die ersten acht Werte für $t, m, \sigma$ an, die (44) bzw. (43) erfüllen.

\begin{tabular}{|r|r|r|r|r|r|r|r|c|}
\hline$n$ & 1 & 2 & 3 & 4 & 5 & 6 & 7 & 8 \\
\hline$t_{n}$ & 1 & 12 & 121 & 1200 & 11881 & 117612 & 1164241 & 11524800 \\
\hline$m_{n}$ & 3 & 25 & 243 & 2401 & 23763 & 235225 & 2328483 & 23049601 \\
\hline$\sigma_{n}$ & 1 & 10 & 99 & 980 & 9701 & 96030 & 950599 & 9409960 \\
\hline
\end{tabular}

Tabelle 2

Es lassen sich folgende Vermutungen beweisen:

$$
\begin{aligned}
& t_{n+1}-\sigma_{n+1}=t_{n}+\sigma_{n} ; \quad m_{n+1}-2 \sigma_{n+1}=m_{n}+2 \sigma_{n}, \\
& \sigma_{n+1}=5 \sigma_{n}+4 t_{n}+1=5 \sigma_{n}+2 m_{n}-1, \\
& t_{n+1}=6 \sigma_{n}+5 t_{n}+1 ; \quad m_{n+1}=12 \sigma_{n}+5 m_{n}-2, \\
& \sigma_{1}=1, \sigma_{2}=10, \sigma_{n+2}=10 \sigma_{n+1}-\sigma_{n}, \\
& t_{1}=1, t_{2}=12, t_{n+2}=10 t_{n+1}-t_{n}+2 ; \\
& \quad m_{1}=3, m_{2}=25, m_{n+2}=10 m_{n+1}-m_{n}-4,
\end{aligned}
$$




$$
\begin{aligned}
& \sigma_{2 n}=2\left(4 t_{n}+1\right) \sigma_{n}=2\left(2 m_{n}-1\right) \sigma_{n}, \\
& t_{2 n}=4\left(2 t_{n}+1\right) t_{n} ; \quad m_{2 n}=\left(2 m_{n}-1\right)^{2} .
\end{aligned}
$$

Es lässt sich zeigen, dass damit die Lösungen von (43) bzw. (44) vollständig erfasst werden. Außerdem gilt nach (46) und (44)

$$
\frac{\sigma_{n+1}}{\sigma_{n}}=5+\frac{4 t_{n+1}}{\sigma_{n}}=5+\sqrt{3} \cdot \frac{4 t_{n+1}}{\sqrt{t_{n}\left(2 t_{n}+1\right)}}=5+\sqrt{3} \cdot \sqrt{8+\frac{1}{t_{n}\left(2 t_{n}+1\right)}}
$$

mit $\lim _{n \rightarrow \infty} \frac{\sigma_{n+1}}{\sigma_{n}}=5+\sqrt{24}$, so dass für $\sigma_{n}$ - wie nach der Rekursionsformel (48) zu erwarten war - nach Berücksichtigung eines normierenden Faktors (vgl. $n=1$ ) eine BINET-Formel entsteht:

$$
\sigma_{n}=\frac{1}{2 \sqrt{24}} \cdot\left((5+\sqrt{24})^{n}-(5-\sqrt{24})^{n}\right) .
$$

Wir kommen nun auf die zweite Teilbarkeitsbedingung $\lambda \mid \alpha s$ mit $\lambda=3, \alpha=2 \gamma-1=$ $4 \delta+1, s=2 \sigma$ zurück. Sie ist genau dann erfüllt, wenn 3 Teiler von $\sigma$ oder Teiler von $4 \delta+1$ ist.

Zum Fall $3 \mid \sigma$ : Nach Tabelle 2 ist $\sigma_{3} \equiv 0 \bmod 3, \sigma_{6} \equiv 0 \bmod 3$, und allgemein führt $\sigma_{3 n^{\prime}} \equiv 0 \bmod 3 \mathrm{zu} \sigma_{3\left(n^{\prime}+1\right)} \equiv 0 \bmod 3$ wegen $\sigma_{3\left(n^{\prime}+1\right)} \equiv \sigma_{3 n^{\prime}+2}-\sigma_{3 n^{\prime}+1} \equiv \sigma_{3 n^{\prime}+1}-$ $\sigma_{3 n^{\prime}+1}-\sigma_{3 n^{\prime}} \equiv 0 \bmod 3$ nach (48). Aus $\sigma_{3 n^{\prime}} \equiv 0 \bmod 3$ folgt nach (48) andererseits $\sigma_{3 n^{\prime}-1} \equiv \sigma_{3 n^{\prime}-2} \bmod 3$, wobei der Rest 0 nicht angenommen wird, weil sonst alle $\sigma_{n}$ durch 3 teilbar wären im Widerspruch zu $\sigma_{1}=1, \sigma_{2}=10$. Es gilt also $3 \mid \sigma_{n}$ genau dann, wenn $3 \mid n$.

Zum Fall $3 \mid 4 \delta+1$ : Aus (44) und (44') ergibt sich $\delta=\sigma+t$ und daher $4 \delta+1=4 \sigma+$ $4 t+1$. Indiziert man die rechte Seite mit $n$, so gelangt man gemäß (46) und (48) zu den äquivalenten Bedingungen $3 \mid \sigma_{n+1}-\sigma_{n}$ und $3 \mid \sigma_{n+2}$.

Insgesamt ist - in indizierter Schreibweise - die zweite Teilbarkeitsbedingung $\lambda \mid \alpha_{n} s_{n}$ mit $\lambda=3$ daher genau dann erfüllt, wenn $n \not \equiv 2 \bmod 3$ ist. Dabei ist $k_{n}=2 m_{n}-1=4 t_{n}+1$, also nach (50)

$$
k_{n}=\frac{\sigma_{2 n}}{2 \sigma_{n}},
$$

und wegen $\mu=2, s_{n}=2 \sigma_{n}$ gilt für $\alpha_{n}$ nach (40), (46): $\alpha_{n}=k_{n}+4 \sigma_{n}=4 t_{n}+1+4 \sigma_{n}$ bzw.

$$
\alpha_{n}=\sigma_{n+1}-\sigma_{n} .
$$

Daraus folgt für $\beta_{n}$ im Hinblick auf (39') wegen $\lambda-\mu=1$ :

$$
\beta_{n}=\sigma_{n+1}+\sigma_{n} .
$$

Die ,quasi-reziproke“ Gleichung (38') ist daher für $s_{n}=2 \sigma_{n}$ und $\alpha_{n}, \beta_{n}$ aus (54) und (55) erfüllt, und für $n \not \equiv 2 \bmod 3$ sind die geforderten Teilbarkeitsbedingungen - trivial: $2 \mid\left(\sigma_{n+1}+\sigma_{n}\right) 2 \sigma_{n}$, nichttrivial: $3 \mid\left(\sigma_{n+1}-\sigma_{n}\right) 2 \sigma_{n}$ - garantiert:

$$
\frac{\left(\sigma_{n+1}-\sigma_{n}\right) \cdot 2 \sigma_{n}}{\left(\sigma_{n+1}+\sigma_{n}\right) \cdot 2 \sigma_{n}}=\frac{2\left(\sigma_{n+1}+\sigma_{n}\right) 2 \sigma_{n}+1}{3\left(\sigma_{n+1}-\sigma_{n}\right) 2 \sigma_{n}+1}
$$


wobei $\frac{\sigma_{n+1}-\sigma_{n}}{\sigma_{n+1}+\sigma_{n}}$ ein vollständig gekürzter Bruch ist, der mit $\frac{\sigma_{2 n}}{2 \sigma_{n}}$ zum Bruch auf der rechten Seite von (56) erweitert wird. Weiterhin gilt nach $\left(37^{\prime}\right) g_{n}=b_{n}\left(2 b_{n}+1\right)$ bzw. (u.a. nach (55))

$$
g_{n}=\left(\sigma_{n+1}+\sigma_{n}\right) \cdot 2 \sigma_{n} \cdot\left(4\left(\sigma_{n+1}+\sigma_{n}\right) \sigma_{n}+1\right)
$$

mit $g_{1}=990=99 \cdot 10, g_{2}=9506980=9701 \cdot 980$, so dass man - vgl. Tabelle $2-$ folgenden vereinfachten Ausdruck für $g_{n}$ vermuten kann:

$$
g_{n}=\sigma_{2 n+1} \cdot \sigma_{2 n}
$$

Zum Nachweis greift man auf die Beziehungen (44), (46), (50) und (51) zurück und stellt fest, dass die rechten Seiten von (57) und (58) mit $5 \sigma_{2 n}^{2}+\frac{1}{2} \cdot \sigma_{4 n}$ übereinstimmen.

Schließlich sind noch $a_{n}^{2}+q_{n}, b_{n}^{2}+r_{n}$ zu bestimmen:

$a_{n}^{2}+q_{n}=\frac{q_{n}}{a_{n}} \cdot g_{n}=\frac{1}{3} g_{n}=\frac{1}{3} \cdot \sigma_{2 n+1} \cdot \sigma_{2 n} ; \quad b_{n}^{2}+r_{n}=\frac{r_{n}}{b_{n}} \cdot g_{n}=\frac{1}{2} g_{n}=\frac{1}{2} \cdot \sigma_{2 n+1} \cdot \sigma_{2 n}$.

Insgesamt gilt nach Satz 3:

Satz 4. Es gibt unendlich viele g-adische Systeme mit 1/1-Darstellungen. Dazu gehören $g_{n}=\sigma_{2 n+1} \cdot \sigma_{2 n}, \sigma_{n}$ aus (52), $n \neq \equiv 2 \bmod 3$, mit den Darstellungen

$$
\left(\left(\sigma_{n+1}-\sigma_{n}\right) \cdot 2 \sigma_{n}, \frac{1}{3} \sigma_{2 n+1} \cdot \sigma_{2 n}\right)_{\sigma_{2 n+1} \cdot \sigma_{2 n}}=\left(\frac{\frac{1}{3} \sigma_{2 n+1} \cdot \sigma_{2 n}}{\left(\sigma_{n+1}-\sigma_{n}\right) \cdot 2 \sigma_{n}}\right)_{\sigma_{2 n+1} \cdot \sigma_{2 n}}
$$

gemeinsamer Wert: $\left(\sigma_{n+1}-\sigma_{n}\right) 2 \sigma_{n} \frac{1}{3}$

$$
\left(\left(\sigma_{n+1}+\sigma_{n}\right) \cdot 2 \sigma_{n}, \frac{1}{2} \sigma_{2 n+1} \cdot \sigma_{2 n}\right)_{\sigma_{2 n+1} \cdot \sigma_{2 n}}=\left(\frac{\frac{1}{2} \sigma_{2 n+1} \cdot \sigma_{2 n}}{\left(\sigma_{n+1}+\sigma_{n}\right) \cdot 2 \sigma_{n}}\right)_{\sigma_{2 n+1} \cdot \sigma_{2 n}}
$$

gemeinsamer Wert: $\left(\sigma_{n+1}+\sigma_{n}\right) 2 \sigma_{n} \frac{1}{2}$.

Beispiele (zu (56) und Satz 4):

$$
\begin{aligned}
n=1: & \frac{9 \cdot 2 \cdot 1}{11 \cdot 2 \cdot 1}=\frac{2 \cdot 11 \cdot 2 \cdot 1+1}{3 \cdot 9 \cdot 2 \cdot 1+1} \quad\left(k_{1}=5\right), \\
& ([18],[330])_{990}=\left(\frac{[330]}{[18]}\right)_{990}=18 \frac{1}{3} ;([22],[495])_{990}=\left(\frac{[495]}{[22]}\right)_{990}=22 \frac{1}{2} ; \\
n=3: & \frac{881 \cdot 2 \cdot 99}{1079 \cdot 2 \cdot 99}=\frac{2 \cdot 1079 \cdot 2 \cdot 99+1}{3 \cdot 881 \cdot 2 \cdot 99+1} \quad\left(k_{3}=485\right), \\
& ([174438],[30428673990])_{91286021970}=\left(\frac{[30428673990]}{[174438]}\right)_{91286021970}=174438 \frac{1}{3}, \\
& ([213642],[45643010985])_{91286021970}=\left(\frac{[45643010985]}{[213642]}\right)_{91286021970}=213642 \frac{1}{2} ; \\
n=4: & \frac{8721 \cdot 2 \cdot 980}{10681 \cdot 2 \cdot 980}=\frac{2 \cdot 10681 \cdot 2 \cdot 980+1}{3 \cdot 8721 \cdot 2 \cdot 980+1} \quad\left(k_{4}=4801\right) .
\end{aligned}
$$

Die Darstellungen gemäß Satz 4 sollen hier übergangen werden. 


\subsection{2 $g$-adische Systeme mit 1/2-Darstellungen ${ }^{4}$}

Es gilt also unendlich viele $g_{n}$ zu finden mit

$$
g_{n}=\frac{a_{n}^{3}}{q_{n}}+a_{n}\left(q_{n}<a_{n}, q_{n} \mid a_{n}^{3}\right) \quad \text { und } g_{n}^{2}=\frac{b_{n}^{3}}{r_{n}}+b_{n}\left(r_{n}<b_{n}, r_{n} \mid b_{n}^{3}\right) .
$$

Hier führt die mit den Bedingungen konforme Spezialisierung $a_{n}=2 \alpha_{n}, q_{n} \mid 4 \alpha_{n}^{2}$, $b_{n}=4 \alpha_{n}^{2}, r_{n}=2 \alpha_{n}^{2} \mathrm{zu}$

$$
\alpha_{n}^{2}=\frac{1}{2} q_{n}\left(q_{n}-1\right) .
$$

Die ersten Beispiele sind $1^{2}=\frac{1}{2} \cdot 2 \cdot 1,6^{2}=\frac{1}{2} \cdot 9 \cdot 8,35^{2}=\frac{1}{2} \cdot 50 \cdot 49,204^{2}=\frac{1}{2} \cdot 289 \cdot 288$. Wegen $q_{n}<2 \alpha_{n}$ muss dabei aber nach (62) $q_{n}>2$ sein. Da $\left(q_{n}\right)$ streng monoton wächst, ist $n=1\left(q_{1}=2\right)$ auszuschließen. Es lässt sich nun zeigen, dass es unendlich viele solcher quadratischer Werte von arithmetischen Reihen gibt: Es gilt die Rekursionsformel $\alpha_{n+2}=6 \alpha_{n+1}-\alpha_{n}$ und

$$
\alpha_{n}=\frac{1}{2 \sqrt{8}} \cdot\left((3+\sqrt{8})^{n}-(3-\sqrt{8})^{n}\right), \quad q_{n}=\frac{1}{2}\left(\sqrt{8 \alpha_{n}^{2}+1}+1\right) \quad(n>1) .
$$

Wegen $g_{n}=2 \alpha_{n}\left(2 q_{n}-1\right)=\alpha_{2 n}$ erhält man schließlich

Satz 5. Es gibt unendlich viele g-adische Systeme mit 1/2-Darstellungen. Dazu gehören $g_{n}=\alpha_{2 n}, \alpha_{n}$ aus (62) bzw. (63), $n>1$, mit den Darstellungen

$$
\begin{aligned}
\left(2 \alpha_{n}, q_{n}\left(2 q_{n}-1\right)\right)_{\alpha_{2 n}}=\left(\frac{q_{n}\left(2 q_{n}-1\right)}{2 \alpha_{n}}\right)_{\alpha_{2 n}} \quad \text { (1 Nachkommastelle) } \\
\left.\left(4 \alpha_{n}^{2},\left[\frac{\alpha_{2 n}}{2}\right][0]\right)_{\alpha_{2 n}}=\left(\frac{\left[\frac{\alpha_{2 n}}{2}\right][0]}{4 \alpha_{n}^{2}}\right)_{\alpha_{2 n}} \quad \text { (2 Nachkommastellen }\right) .
\end{aligned}
$$

\section{Beispiele:}

$n=2:([12],[153])_{204}=\left(\frac{[153]}{[12]}\right)_{204}\left(\right.$ gemeinsamer Wert: $\left.12 \frac{3}{4}\right)$,

$([144],[102][0])_{204}=\left(\frac{[102][0]}{[144]}\right)_{204}\left(\right.$ gemeinsamer Wert: $\left.144 \frac{1}{2}\right)$;

$n=3:([70],[4950])_{6930}=\left(\frac{[4950]}{[70]}\right)_{6930}\left(\right.$ gemeinsamer Wert: $\left.70 \frac{5}{7}\right)$,

$([4900],[3465][0])_{6930}=\left(\frac{[3465][0]}{[4900]}\right)_{6930}\left(\right.$ gemeinsamer Wert: $\left.4900 \frac{1}{2}\right)$.

\footnotetext{
${ }^{4}$ In diesem Abschnitt wird aus Platzgründen auf die Beweise verzichtet. Die Methoden ähneln denen in 2.3.1.
} 
Bemerkung: Es lassen sich auch Einzelbeispiele mit 1/2-Darstellungen konstruieren, bei denen die zweite Nachkommastelle ,besetzt“" ist, z.B.

$$
\begin{aligned}
& ([6214],[38614134])_{709906002}=\left(\frac{[38614134]}{[6214]}\right)_{709906002}\left(\text { gemeinsamer Wert:6214 } \frac{13}{239}\right), \\
& ([38613796],[2100313][436865232])_{709906002}=\left(\frac{[2100313][436865232]}{[38613796]}\right)_{709906002} \\
& \left(\text { gemeinsamer Wert: } 38613796 \frac{1}{338}\right) .
\end{aligned}
$$

Ein Nachweis dafür, dass unendlich viele 1/2-Darstellungen mit „besetzten“ zweiten Nachkommastellen existieren, konnte nicht geführt werden.

\section{Weitere Fragen}

zu Satz 3:

Gibt es $M / N$-Darstellungen mit (o.B.d.A.) $M \leq N$ sowie $(M, N) \neq(1,1),(1,2)$ ?

Gibt es insbesondere 2/2-Darstellungen und solche, die sich auf mehr als 2 Nachkommastellen $(n \geq 2)$ beziehen?

Gibt es ein praktikables Kriterium, das im Fall $g=\frac{a^{3}}{q}+a\left(1 \leq q<a, q \mid a^{3}\right)$ weitere Darstellungen ausschließt? (Vgl. die Ausführungen zu $g=10$ in 1.)

\section{zu 2.3.1:}

Lassen sich aus (37) mit $q \nmid a \vee r \nmid b$ unendlich viele $g$ mit 1/1-Darstellungen konstruieren? (Vgl. die Beispiele 520 und 5460 in Tabelle 1.)

Schreibt man (39) in der Form $(\beta-\alpha) h=(\lambda-\mu) s$ und vermeidet die Spezialisierung $h=1\left(39^{\prime}\right)$, so erhält man

$$
k+\lambda s=\beta h, \quad k+\mu s=\alpha h, \quad k^{2}-h=\mu \lambda s^{2}, \quad \mu s=\frac{\alpha^{2} h-1}{\alpha+\beta}, \quad \lambda s=\frac{\beta^{2} h-1}{\alpha+\beta} .
$$

Erreicht man auf diese Weise mithilfe der Variablen $h$ einen größeren „Gestaltungsspielraum“, um unendlich viele $g$ mit 1/1-Darstellungen konstruieren zu können?

\section{zu Satz 5:}

Gibt es unendlich viele $g$-adische Systeme mit 1/2-Darstellungen und „besetzten“ zweiten Nachkommastellen?

Stefan Deschauer

Technische Universität Dresden

Fachrichtung Mathematik

D-01062 Dresden, Deutschland

e-mail: Stefan.Deschauer@tu-dresden.de 